\title{
The National Gene Vector Biorepository: Eleven Years of Providing Resources to the Gene Therapy Community
}

\author{
Kenneth Cornetta, ${ }^{1,2 *}$ Lorraine Matheson, ${ }^{1}$ Ryan Long, ${ }^{3}$ and Lisa Duffy ${ }^{1}$ \\ ${ }^{1}$ Department of Medical and Molecular Genetics, ${ }^{2}$ Brown Center for Immunotherapy, and ${ }^{3}$ University Information Technology Services, \\ Indiana University School of Medicine, Indianapolis, Indiana.
}

The National Gene Vector Biorepository (NGVB) program has been highly accessed by gene therapy investigators. The reagent repository has distributed over 1,000 reagents to 397 investigators. The Pharmacology/Toxicology Archive contains over 36,000 specimens from a variety of adeno-associated virus (AAV), adenoviral, and other pharmacology/toxicology studies. NGVB also maintains a searchable database of gene therapy pharmacology/toxicology studies to promote data sharing. NGVB has provided Food and Drug Administration (FDA)-mandated replication-competent virus testing for over 70 clinical trials. From 2008 to 2018, there have been 114 publications acknowledging the NGVB. It is unlikely that any other National Institutes of Health (NIH)-funded program has served as many gene therapy investigators as the NGVB.

Keywords: gene therapy resources, FDA required testing, Pharm/Tox studies, reagent repository

\section{INTRODUCTION}

THE AIM OF the National Gene Vector Biorepository (NGVB) is to assist gene therapy investigators by addressing barriers to clinical implementation of their research. As depicted in Fig. 1, services include access to novel reagents and assistance with meeting the unique Food and Drug Administration (FDA) requirements of gene therapy preclinical and clinical studies.

This National Institutes of Health (NIH) program was initially funded in 2008 by the National Center for Research Resources (NCRR) as an Animal Model and Animal and Biological Material Resource (P40) Grant. The program was available to investigators eligible to apply for NIH funding. A competitive renewal was funded just before the disbanding of the NCRR in December 2011. While initially slated to become part of the newly formed National Center for Advancing Translational Science (NCATS), the National Heart, Lung, and Blood Institute (NHLBI) opted to bring the program under its portfolio. In 2018, the NHLBI elected to convert the NGVB funding mechanism from a P40 grant to a contract and renew a revised program for another 5 years.

\section{RESOURCES AND SERVICES}

The NGVB has been charged with providing a varied array of services and resources to gene therapy investi- gators. The program is based within the Indiana University School of Medicine. The activities are governed by the NGVB Policy and Procedure Manual, which is available on the NGVB website (www.NGVBCC.org). Under the grant mechanism, eligible investigators were those who (1) were eligible to receive NIH support; (2) agree to comply with applicable NIH and FDA regulations and those of their local Institutional Biosafety Committee and Institutional Review Board; (3) agree to assurance and certification requirements as described in the Application for a Public Health Service Grant; and (4) disclose grant support for all research projects using NGVB services. Investigators must also agree to acknowledge the NGVB in all publications. NGVB personnel do not expect authorship unless the service requested required assay development or other specialized work. During the grant period, an external advisory board (EAB) assisted in oversight of the program and included experts in gene therapy and representatives from the NIH, FDA, and the Office of Biotechnology Activities.

Access to NGVB services is made possible through the NGVB website (Fig. 2). The site hosts a number of publicly available educational resources, while services require registration, which is done through the website. In the following sections, the specifics of each service area are described along with information about past usage.

\footnotetext{
${ }^{*}$ Correspondence: Dr. Kenneth Cornetta, Department of Medical and Molecular Genetics, 980 W. Walnut St. R3 C649, Indianapolis, IN 46202. E-mail: kcornett@iu.edu
} 


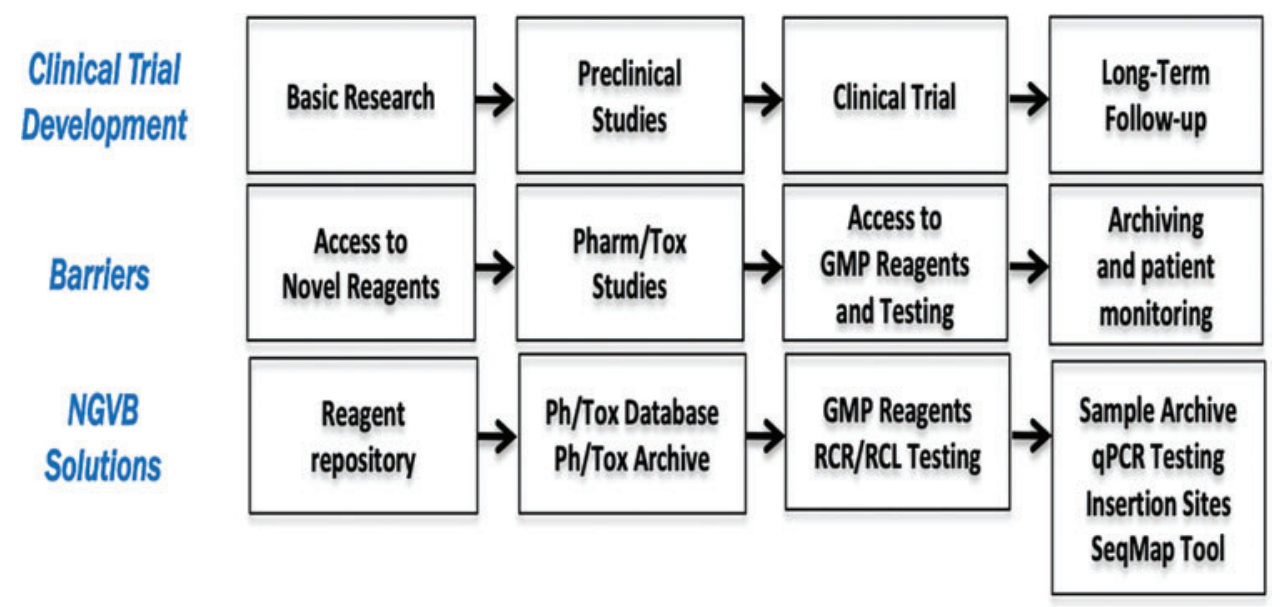

Figure 1. NGVB Resources Address Barriers to Clinical Gene Therapy Development. The NGVB provides resources that span from basic gene therapy research to clinical trial monitoring. NGVB, National Gene Vector Biorepository. Color images are available online.

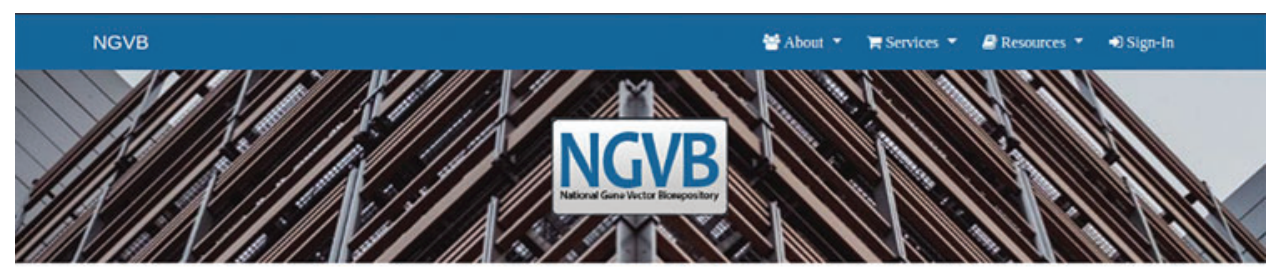

The National Gene Vector Biorepository (NGVB)

We are an NHLBI/NIH funded resource for investigators conducting clinical gene therapy research.

Our databases and educational resources are open to everyone. Other services may be limited to academic institutions, non-profit organizations, and companies that quality for $\mathrm{NIH}$ small-business grants.

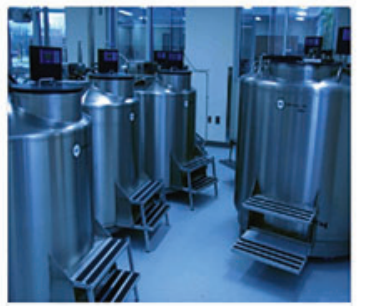

Archiving

The NGVB can provide GLP archiving of samples generated for Pharm/Tox studies. Storage of GMP material and clinical samples is also available. The services are provided free for eligible institutions.

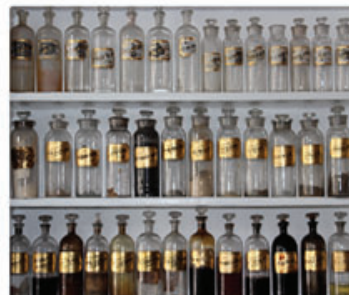

Reagent Repository

The NGVB has a repository of over 80 reagents, including $A A V$ and lentiviral vector plasmids, certified cell lines, and retroviral packaging cell lines.

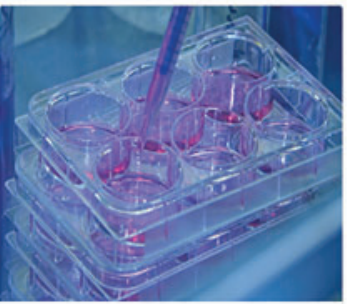

\section{Testing}

The FDA requires testing of vector products, gene modified cells, as well as post-trial monitoring of patients treated with gene therapy. The NGVB provides an array of tests to help investigators meet these unique FDA requirements.

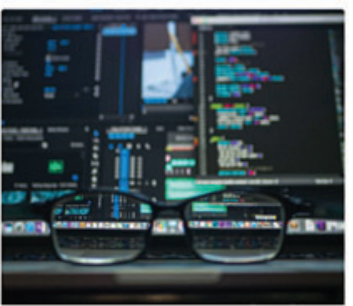

Seqmap 3

SeqMap 3 is a publically available biointormatics tool suitable for identifying vector insertion sites from Next-Gen Sequencing of LAM.PCR and nrLAM.PCR

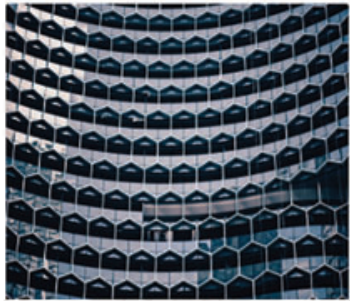

Pharm/Tox Database

The NGVB provides detaled summaries of over $\mathbf{5 0}$ gene therapy Pharmacology and Toxicology studies. We can tacilitate letters of cross-reterence so investigators can avoid repeating studies alreacy on flie with the FDA.

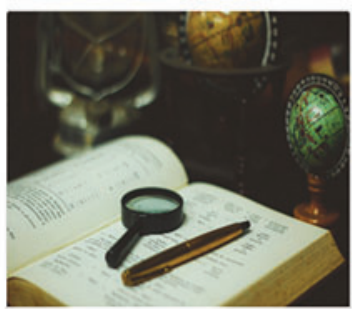

Educational Resources

This website provides links to funding agencies, NIH resources, gene therapy societies, and important FDA documents.

Figure 2. NGVB Home Page. The home page provides an overview of NGVB resources and services and can be found at www.NGVBCC.org Color images are available online. 


\section{Repository}

The NGVB website currently contains a list of 93 unique reagents, including plasmids for adeno-associated virus (AAV) gene transfer (provided by the University of North Carolina) and cell lines used in manufacture of retroviral, lentiviral, adenoviral, and AAV gene therapy. The NGVB can store the adenovirus reference standard that was initially developed through the collaborative efforts of the FDA, Williamsburg BioProcessing Foundation (WilBio), and members of academia and industry. ${ }^{1}$ When WilBio was closed, the NGVB was selected as the site to house this reagent so that it would continue to be made available to the gene therapy community.

The NGVB website has a searchable catalog of reagents with information about the biosafety level, sequence, or cell line derivation, along with relevant publications. Reagents are requested by adding them to a cart, similar to commercial ordering sites. Once a reagent is requested and eligibility confirmed, the NGVB emails a material transfer agreement (MTA) and contact information for the institution depositing the reagent. After the MTA is completed, the reagent is shipped. To date, the reagent repository has facilitated the distribution of 1,077 reagents to 397 investigators (Table 1). Cost saving is a particular advantage of maintaining the cell banks within NGVB, a number of which have undergone the FDA-required testing for clinical manufacturing. Use of these lines may limit the number of release testing methods required for investigators planning on generating clinical products with these cell lines, with an estimated cost savings of \$60-90,000. The archive also helps investigators by depositing reagents in the repository. NIH expects reagent sharing, even after a grant has expired, which can incur significant expense in regenerating stocks of popular reagents. The NGVB assists in reagent sharing while allowing investigators to focus on their current research efforts.

\section{Pharmacology and toxicology (Pharm/Tox studies) good laboratory practice archive}

When an investigator performs a biodistribution or toxicity study in support of an investigational new drug (IND), the FDA has specific requirements for archiving the samples in case additional analyses are required (CFR 21 CFR 58). The vast majority of NHLBI investigators lack the space, quality assurance infrastructure,

Table 1. Reagents by type and usage currently in the National Gene Vector Biorepository reagent repository

\begin{tabular}{lcc}
\hline Reagent Type & Number in Repository & Number Shipped \\
\hline Antibody & 1 & 2 \\
Reference standard & 1 & 1 \\
Vector & 17 & 5 \\
Cell line & 21 & 333 \\
Plasmid & 53 & 703 \\
Total & 93 & 1,044 \\
\hline
\end{tabular}

and experience to maintain a good laboratory practice (GLP) archive, placing them at risk for noncompliance with FDA requirements. Since samples must be stored for a minimum of 5 years, the time required for storage generally exceeds the period of funding for the grant. Cost saving is another advantage. Using the Indiana Clinical and Translational Sciences sample storage facility, construction of which was paid for in part by an NIH construction grant, the archived material can be stored in a controlled environment with $24 / 7$ monitoring and purposefully designed electrical backup at $5-10 \%$ of the cost of a commercial storage facility. The facility standard operating procedures (SOPs) are available online (https://indianactsi.org/researchers/services-tools/ biospecimen-services/biomanagement/specimen-storagefacility-standard-operating-procedures/) and complement NGVB SOPs that oversee GLP compliance for monitoring, auditing, receipt, and destruction of archived samples. At this writing, the NGVB maintains 36,527 samples from $21 \mathrm{NIH}$-funded GLP studies. The number and type of samples are shown in Fig. 3. NGVB also offers a clinical sample archive given the FDA requirement for 15 years of patient monitoring. ${ }^{2}$

\section{Pharm/Tox database}

The Pharm/Tox database contains an online database of animal safety studies submitted to the U.S. FDA in support of an IND application. ${ }^{3}$ The results are used to determine if an IND application may proceed to clinical trial. These studies typically are done under GLP guidelines and may involve a variety of animal species, from mice to nonhuman primates. Increased awareness of these studies is also important given the expense involved (mouse and rabbit studies in the Pharm/Tox database ranged from $\$ 541,750$ to $\$ 619,701$ per study and an NHLBI-funded porcine study was $\$ 1.2$ million).

Data submitted in an IND are confidential, and the FDA cannot apply safety data submitted by one investigator to the benefit of another without specific permission (i.e., Letter of Cross Reference). The Pharm/Tox can therefore foster data sharing in Pharm/Tox studies. In addition, given that most gene therapy investigators have little experience with Pharm/Tox studies, the database can also serve as an educational tool to illustrate Pharm/Tox study design. The database currently contains 52 studies, the majority of which were evaluating AAV vectors in a variety of animal models.

\section{Replication-competent virus testing}

Gene therapy has a number of unique regulatory requirements for clinical trials using retroviral and lentiviral vectors, including testing for replication-competent retrovirus (RCR) and replication-competent lentivirus (RCL) ${ }^{2,4-6}$ Although the majority of integrating vectors utilized in clinical trials are designed to be replication defective, older manufacturing methods were prone to 


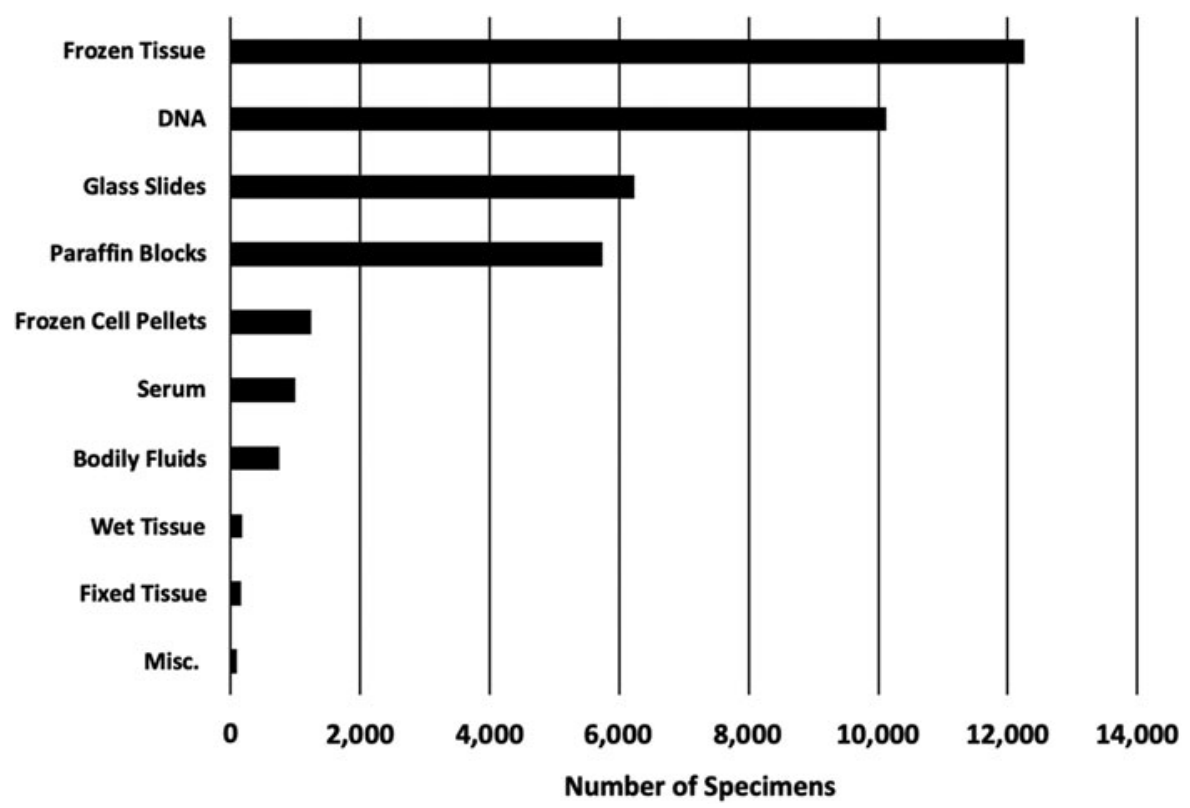

Figure 3. Current Number of Samples Stored in the NGVB Pharmacology/Toxicology Archive. The NGVB stores a variety of samples in compliance with U.S. Food and Drug Administration Good Laboratory Practice archiving guidelines.

recombination leading to development of $\mathrm{RCR}^{7,8}$ The concern for inadvertent exposure to RCR and RCL was significantly heightened after the development of leukemia in patients treated with the retroviral vector. The number of integrations per cell appears to be one of a number of complex factors, leading to malignant transformation. ${ }^{9-11}$ While patients who developed malignancies related to vector insertion had no evidence of RCR, an active virus infection would likely cause multiple integration events that would increase the change for insertional mutagenesis and malignancy.

The NGVB has offered screening of vector-transduced cells for RCR and RCL. The assay conforms to the published FDA specifications, which include testing $1 \%$ (up to a maximum of $10^{8}$ ) transduced cells. Indiana University had previously developed a number of RCR and RCL assays that take into account the unique aspects of each vector type, including the vector pseudotype..$^{7,12-14}$ The guidance also specifies that cells must be passaged for a minimum of five passages (typically 21 days) followed by analysis for RCR/RCL, in part, because the growth rate of any recombinant virus will be unknown at the time of testing. To minimize receptor interference by vector particles, the assay requires a large number of cells for virus amplification, which greatly impacts the assay cost.

The NGVB has published two compilation articles based on screening ex vivo transduced cell products. One article provided data on 282 retroviral vectortransduced cell productions from 16 clinical retroviral trials infused into 270 patients. ${ }^{15}$ The other screened
$\mathrm{T}$ cell products from 26 clinical trials, totaling 460 transduced $\mathrm{T}$ cell products from 375 subjects. ${ }^{16}$ To date, all analyses of ex vivo treated cells have been negative for RCR and RCL. Given that all cell products tested were exposed to a vector previously screened negative for RCR or RCL, it appears that testing the transduced products does not add value if the manufactured vector is RCR/RCL free. One caveat is that clinical trials utilized retroviral vectors generated in the PG13 cell line or third-generation lentiviral vectors manufactured by the transient transfection method; to date, no RCR or RCL has been detected using these manufacturing methods. Testing cell products may be required if few well-characterized methods of manufacturing are utilized.

NGVB also offers qPCR testing of post-trial blood samples for RCR or RCL, targeting the vector envelope sequences. Currently, testing is required for vector products and ex vivo transduced cells. In addition, blood monitoring for RCR/RCL is required pretreatment, at 3, 6 and 12 months. At this writing, 1,735 retroviral and 461 lentiviral samples have screened negative. The samples were collected at various time points after exposure and were collected from 76 clinical trials. These data should provide additional support for U.S. FDA draft guidance "Testing of Retroviral Vector-Based Human Gene Therapy Products for Replication Competent Retrovirus During Product Manufacture and Patient Follow-up," which may decrease the frequency and extent of postinfusion monitoring for RCR/RCL. As of this writing, this draft FDA guidance document has not been finalized. 


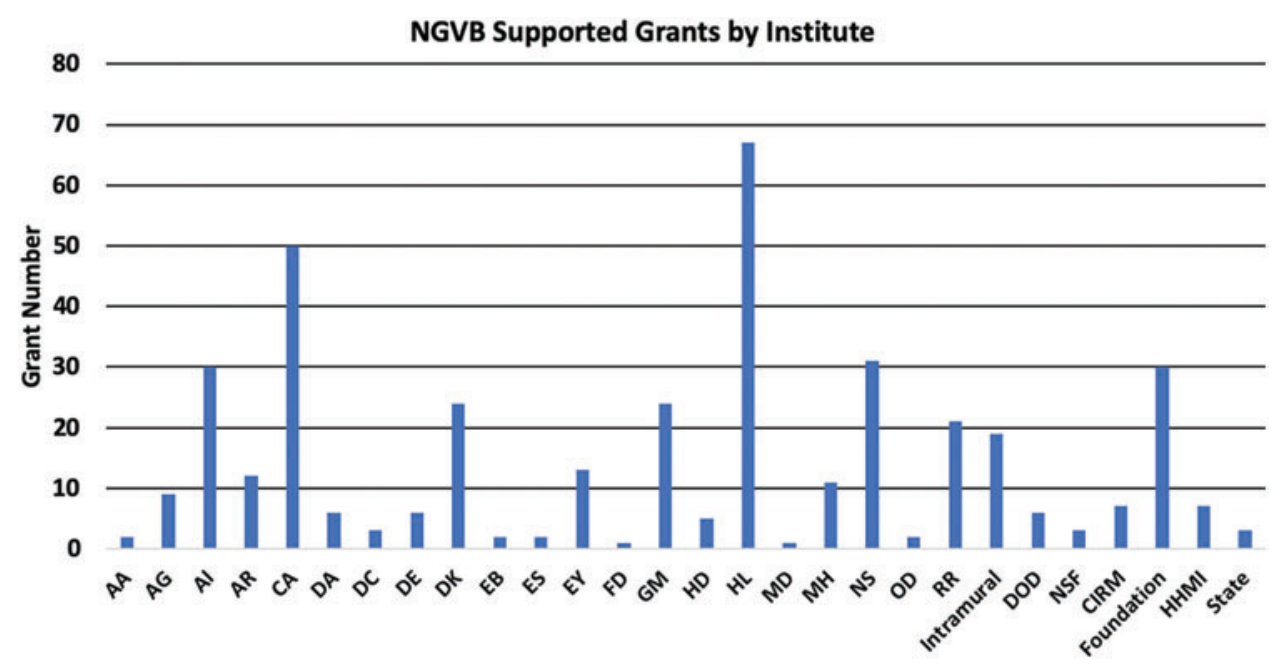

Figure 4. The Number of Grants Supported by NGVB. The numbers of grants are organized by the NIH or other granting organizations. The two-letter acronyms represent NIH institutes and Centers (see https://grants.nih.gov/grants/acronym_list.htm for key). Intramural, Intramural NIH investigators; DOD, Department of Defense; NSF, National Science Foundation; CIRM, California Institute for Regenerative Medicine; Foundation, not-for-profit foundations (ex. American Cancer Society); HHMI, Howard Hughes Medical Institutes; State, funding from a U.S. State entity. NIH, National Institutes of Health. Color images are available online.

\section{Going forward}

In review of the first 10 years of the program, we noted that investigators from every NIH institute received support for testing, archiving, or material from the reagent repository (Fig. 4). With the awarding of the NGVB contract in December of 2018, current services will still be available and expanded to include vector copy number and insertion site testing (in collaboration with the DanaFarber Cancer Institute). The eligibility requirements have changed in the new contract and certain testing and archiving services may be restricted. Investigators seeking these services are encouraged to contact the NGVB to confirm eligibility on any new project.

\section{CONCLUSION}

The rationale for offering NGVB services includes (1) decreasing the risk of noncompliance with FDA requirements by providing investigators with GLP and GMP services; (2) addressing the disconnection between clinical trial grant funding periods and the extended post-trial monitoring requirements of the FDA; and (3) providing the opportunity for reducing costs. A cost savings example is our published study of 460 CAR-T cell clinical products for RCL. This is estimated at $\$ 4$ million given the average commercial cost of $\$ 16,500$ per RCL assay. Furthermore, by centralizing FDA-required RCR and RCL testing, the ability to summarize results across a large number of clinical trials is providing regulators, clinical trial reviewers, scientists, and clinicians with important safety data that can inform the risk: benefit ratio for gene therapy. In summary, NGVB services have been heavily utilized and impacted investigators funded by every NIH institute. While the NGVB contract has altered eligibility for some services, there will be an expansion of services for eligible gene therapy investigators.

\section{ACKNOWLEDGMENTS}

The authors wish to thank the NGVB technicians Sue Koop, Jing Yao, Emily Nance, Kimberley House, Chelsey Lamm, and Stephanie Baker.

\section{AUTHOR DISCLOSURE}

No competing financial interests exist.

\section{FUNDING INFORMATION}

The results discussed in this article were funded under grant numbers P40 RR024928 and 9P40HL116242. Writing of the manuscript occurred during the NGVB contract, which requires the inclusion of the following statement "This project has been funded, in whole or in part, with Federal funds from the National Heart, Lung, and Blood Institute, National Institutes of Health, Department of Health and Human Services, under Contract No. 75N92019D00018." Some biospecimens are stored in the CTSI Specimen Storage Facility, construction of which was funded, in part, by grant number NIH/NCRR RR020128. 


\section{REFERENCES}

1. Palmer DJ, Ng P. Physical and infectious titers of helper-dependent adenoviral vectors: a method of direct comparison to the adenovirus reference material. Mol Ther 2004;10:792-798.

2. United States Food and Drug Administration. Guidance for Industry Gene Therapy Clinical TrialsObserving Subjects for Delayed Adverse Events. November 2006.

3. Cornetta K, Matheson L. The National Gene Vector Biorepository's Pharm/Tox Database. Mol Ther 2009;17:582-584.

4. United States Food and Drug Administration. Guidance for Human Somatic Cell Therapy and Gene Therapy. March, 1998.

5. United States Food and Drug Administration. Content and Review of Chemistry, Manufacturing, and Control (CMC) Information for Human Gene Therapy Investigational New Drug Applications. Guidance for FDA Reviewers and Sponsors. April 2008

6. United States Food and Drug Administration. Guidance for Industry-Supplemental Guidance on Testing for Replication Competent Retrovirus in Retroviral Vector Based Gene Therapy Products and During Follow-up of Patients in Clinical Trials Using Retroviral Vectors. November 2006.
7. Cornetta K, Nguyen N, Morgan RA, et al. Infection of human cells with murine amphotropic replicationcompetent retroviruses. Hum Gene Ther 1993;4: 579-588.

8. Cornetta K, Wilson CA. Safety of retroviral vectors: regulatory and technical considerations. In: Dropulic B, Carter B, eds. Concepts in Genetic Medicine. Hoboken, NJ: John Wiley \& Sons, Inc., 2008:277-288.

9. Hacein-Bey-Abina S, von Kalle C, Schmidt M, et al. LMO2-associated clonal T cell proliferation in two patients after gene therapy for SCID-X1. Science 2003;302:415-419. Erratum: Science 2003;2302, 2568.

10. Ott MG, Schmidt M, Schwarzwaelder K, et al. Correction of $X$-linked chronic granulomatous disease by gene therapy, augmented by insertional activiation of MDS1-EVI1, PRDM16 or SETBP1. Nat Med 2006;12:401-409.

11. Braun CJ, Boztug K, Paruzynski A, et al. Gene therapy for Wiskott-Aldrich syndrome-long-term efficacy and genotoxicity. Sci Transl Med 2014;6: 227ra233.

12. Chen J, Reeves L, Cornetta K. Safety testing for replication-competent retrovirus (RCR) associated with Gibbon Ape Leukemia Virus pseudotyped retroviral vectors. Hum Gene Ther 2001;12:61-70.

13. Duffy L, Koop S, Fyffe J, et al. Extended S+/Lassay for detecting replication competent retroviruses (RCR) pseudotyped with the RD114 viral envelope. Preclinica 2003;May/June: 53-59.

14. Sastry L, Xu J, Johnson T, et al. Certification assays for HIV-1-based vectors: frequent passage of gag sequences without evidence of replication competent viruses. Mol Ther 2003;8: 830-839.

15. Cornetta K, Duffy L, Turtle CJ, et al. Absence of replication-competent lentivirus in the clinic: analysis of infused T cell products. Mol Ther 2018; 26:280-288.

16. Cornetta K, Duffy L, Feldman SA, et al. Screening clinical cell products for replication competent retrovirus: the National Gene Vector Biorepository Experience. Mol Ther Methods Clin Dev 2018;10: 371-378.

Received for publication November 4, 2019; accepted after revision December 18, 2019

Published online: January 6, 2020 